\title{
Modeling large strain electrostriction of ferroelectrics under combined electromechanical loads
}

\author{
Wei Zhang and Kaushik Bhattacharya ${ }^{*}$ \\ Division of Eng. and Applied Science, California Institute of Technology, Pasadena, CA 91125
}

\begin{abstract}
A computational model for investigating domain switching and macroscopic electromechanical properties of ferroelectric materials is developed. Various aspects of domain nucleation and growth, and their effects on macroscopic hysteresis are examined. The model is validated against recent experimental observations. It is thus validated as a design tool to investigate various aspects of a novel thin film ferroelectric microactuator in future work.
\end{abstract}

Keywords: Ferroelectric, model, simulation, actuation strain, electromechanical load, domain switching, hysteresis

\section{INTRODUCTION}

Ferroelectric thin films are currently being extensively investigated for use in microelectromechanical systems (MEMS) as actuators and sensors. One unique characteristic of ferroelectric materials is that their macroscopic behavior strongly depends on their microstructural change in response to external loads. Understanding the microstructural change in a ferroelectric material is hence an important subject for understanding the macroscopic response and for optimizing the design of small devices.

During the past decade, considerable research has been carried out on typical ferroelectric materials to study the relationship between their microstructural evolution and macroscopic properties. In these investigations, the LandauGinzburg-Devonshire and Time Dependent Ginzburg-Landau (TDGL) phenomenological models have been successfully utilized to analyze domain formation in the cubic to tetragonal phase transition process. ${ }^{1-3}$ Yet questions remain concerning domain nucleation and domain switching in ferroelectrics subject to electrical and mechanical loads.

Recently, Burcsu, Zhang, Ravichandran and Bhattacharya have experimentally studied the correlation of the macroscopic properties including hysteresis and the change of microstructures in barium titanate $\left(\mathrm{BaTiO}_{3}\right)$ and lead titanate $\left(\mathrm{PbTiO}_{3}\right)$ under both mechanical and electrical loads. ${ }^{4,5} \mathrm{In}$-situ experimental observations confirmed that domain switching is a fundamental mechanism in large strain actuation. In their experiments, compressive stress and alternating electric field were applied to single-domain specimens to obtain a combination of $90^{\circ}$ and $180^{\circ}$ domain switching. The compressive stress and electric field were systematically varied and an optimal combination of electromechanical loads was found to yield the large actuation strain through $90^{\circ}$ domain switching. In addition, the damage development and fatigue growth were also examined.

In this work, we introduce a computational model based on the formulation of Shu and Bhattacharya ${ }^{6}$ to describe the temporal evolution of domains under electromechanical loading. This is similar to the TDGL model, but treats the electric field explicitly to enable better connection with experiment. We also introduce defects to enable domain nucleation as opposed to adding stochastic noise.

\footnotetext{
* Corresponding author. Tel.: +1-626-395-8306; fax: +1-626-568-2719.

E-mail address: bhatta@caltech.edu (K. Bhattacharya).
} 


\section{MODEL}

Consider a ferroelectric in the shape of a flat plate with electrodes on top and bottom, and uniform dead load corresponding to stress $\sigma_{0}$. The total free energy in the ferroelectric system with volume $\Omega$ can be written as ${ }^{6}$

$$
\mathscr{E}=\int_{\Omega}\left(U\left(p_{i, j}\right)+W\left(\varepsilon_{i j}, p_{i}\right)-\sigma_{0 i j} \varepsilon_{i j}+\frac{\epsilon_{0}}{2} \phi_{, i} \phi_{, i}\right) d \Omega
$$

Here, $U$ is the domain wall energy density (per unit volume) associated with polarization gradient $\nabla \mathbf{p}, W$ the internal energy density due to elastic strain $\varepsilon$ and polarization $\mathbf{p}, \epsilon_{0}|\nabla \phi|^{2} / 2$ the electrostatic energy density associated with polarization $\mathbf{p}$ through Gauss' equation $p_{i, i}-\epsilon_{0} \phi_{, i i}=0$, and $\epsilon_{0}\left(=8.854 \times 10^{-12} \mathrm{~F} / \mathrm{m}\right)$ is the vacuum permittivity. Considering the gradient flow of the energy in Eq. (1), we obtain the governing differential equations

$$
\begin{aligned}
& \mu \dot{p}_{i}=\left(\frac{\partial U}{\partial p_{i, j}}\right)_{, j}-\frac{\partial W}{\partial p_{i}}-\phi_{, i}, \\
& p_{i, i}-\epsilon_{0} \phi_{, i i}=0, \\
& \left(\frac{\partial W}{\partial \varepsilon_{i j}}\right)_{, j}=\sigma_{i j, j}=0,
\end{aligned}
$$

where $\mu$ represents the mobility. Specializing to two dimensions assuming that the third is infinite, we obtain a state of plane strain and plane polarization. We select the energy functions

$$
\begin{aligned}
U\left(p_{i, j}\right)= & \frac{a_{0}}{2}\left(p_{1,1}^{2}+p_{1,2}^{2}+p_{2,1}^{2}+p_{2,2}^{2}\right), \\
W\left(p_{i}, \varepsilon_{j k}\right)= & \frac{a_{1}}{2}\left(p_{1}^{2}+p_{2}^{2}\right)+\frac{a_{2}}{4}\left(p_{1}^{4}+p_{2}^{4}\right)+\frac{a_{3}}{2} p_{1}^{2} p_{2}^{2}+\frac{a_{4}}{6}\left(p_{1}^{6}+p_{2}^{6}\right)+\frac{a_{5}}{4} p_{1}^{4} p_{2}^{4} \\
& -\frac{b_{1}}{2}\left(\varepsilon_{11} p_{1}^{2}+\varepsilon_{22} p_{2}^{2}\right)-\frac{b_{2}}{2}\left(\varepsilon_{11} p_{2}^{2}+\varepsilon_{22} p_{1}^{2}\right)-b_{3} \varepsilon_{12} p_{1} p_{2} \\
& +\frac{c_{1}}{2}\left(\varepsilon_{11}^{2}+\varepsilon_{22}^{2}\right)+c_{2} \varepsilon_{11} \varepsilon_{22}+\frac{c_{3}}{2} \varepsilon_{12}^{2}
\end{aligned} .
$$

Note that $W$ is the Landau-Devonshire energy but augmented with an $8^{\text {th }}$ order term (with coefficient $a_{5}$ ). The relationship between polarizations and elastic strains can be established at any given stress state. For example, a stress free state $\left(\sigma_{11}=\sigma_{22}=\sigma_{12}=0\right)$ leads to

$$
\begin{aligned}
& \varepsilon_{11}=\frac{\left(c_{1} b_{1}-c_{2} b_{2}\right) p_{1}^{2}+\left(c_{1} b_{2}-c_{2} b_{1}\right) p_{2}^{2}}{2\left(c_{1}^{2}-c_{2}^{2}\right)} \\
& \varepsilon_{22}=\frac{\left(c_{1} b_{2}-c_{2} b_{1}\right) p_{1}^{2}+\left(c_{1} b_{1}-c_{2} b_{2}\right) p_{2}^{2}}{2\left(c_{1}^{2}-c_{2}^{2}\right)} \\
& \varepsilon_{12}=\frac{b_{3} p_{1} p_{2}}{c_{3}}
\end{aligned}
$$


We normalize the variables as $x_{i}^{\prime}=x_{i} \sqrt{c_{0} / a_{0}} / p_{0}, p_{i}^{\prime}=p_{i} / p_{0}, \mu^{\prime}=1, t^{\prime}=t c_{0} / \mu p_{0}^{2}, \epsilon_{0}^{\prime}=\epsilon_{0} c_{0} / p_{0}^{2}, \phi^{\prime}=\phi / \sqrt{a_{0} c_{0}}$, $a_{0}^{\prime}=1, \quad a_{1}^{\prime}=a_{1} p_{0}^{2} / c_{0}, \quad a_{2}^{\prime}=a_{2} p_{0}^{4} / c_{0}, \quad a_{3}^{\prime}=a_{3} p_{0}^{4} / c_{0}, \quad a_{4}^{\prime}=a_{4} p_{0}^{6} / c_{0}, a_{5}^{\prime}=a_{5} p_{0}^{8} / c_{0}, b_{i}^{\prime}=b_{i} p_{0}^{2} / c_{0}$, and $c_{i}^{\prime}=c_{i} / c_{0}$, where $i=1,2,3$.

If $c_{0}=1 \mathrm{GPa}$ (to obtain reasonable stress and energy barrier) and $p_{0}=0.26 \mathrm{C} / \mathrm{m}^{2}$ (the spontaneous polarization of $\mathrm{BaTiO}_{3}$ ), then the normalized spontaneous polarization becomes unity and the vacuum permittivity is scaled to $\epsilon_{0}^{\prime}=0.131$. For $\mathrm{BaTiO}_{3}$, the dimensionless elastic moduli are chosen as $c_{1}^{\prime}=185, c_{2}^{\prime}=111$ and $c_{3}^{\prime}=54$. The normalized coupling parameters $b_{1}^{\prime}=1.4282, b_{2}^{\prime}=-0.185$ and $b_{3}^{\prime}=0.5886$ are used. In our model, we take $a_{1}^{\prime}=-0.007, a_{2}^{\prime}=-0.009, a_{3}^{\prime}=0.003, a_{4}^{\prime}=0.0261$ and $a_{5}^{\prime}=5$ for barium titanate. These parameters yield energy wells at positions where normalized polarization and strains take the spontaneous values, i.e. $p_{0}^{\prime}=1, \varepsilon_{a}=-0.0044$ and $\varepsilon_{c}=0.0065$.

The contour of the normalized free energy $\mathrm{W}^{\prime}$ as a function of polarization $p_{1}^{\prime}$ and $p_{2}^{\prime}$ is presented in Fig. 1(a). Four energy wells (corresponding to the four crystallographically equivalent invariants in the two dimensional case) are clearly seen. The profile of $\mathrm{W}^{\prime}$ against $p_{1}^{\prime}$ (at $p_{2}^{\prime}=0$ ) is shown in Fig. 1(b). In our simulations, we consider a rectangular computational cell that is composed of $160 \times 40$ discrete grid points (grid size $\Delta x_{i}^{\prime}=5$ ) along horizontal and vertical directions. The computational cell and boundary conditions are illustrated in Fig. 1(c). It should be emphasized that periodic strain instead of periodic displacement boundaries are used. The stress $\sigma_{11}^{\prime}=0$ is assumed on the periodic boundaries. More details about the model and simulation results are available elsewhere. ${ }^{7}$

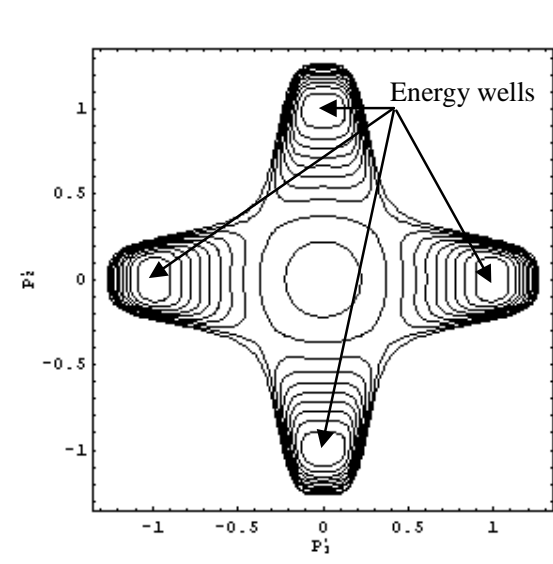

(a)

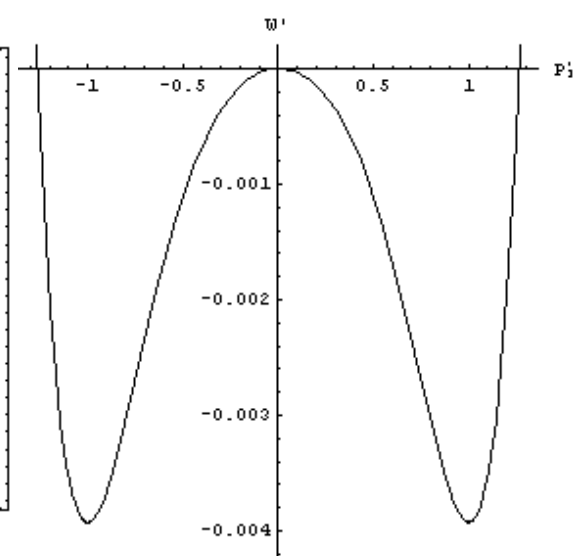

(b)

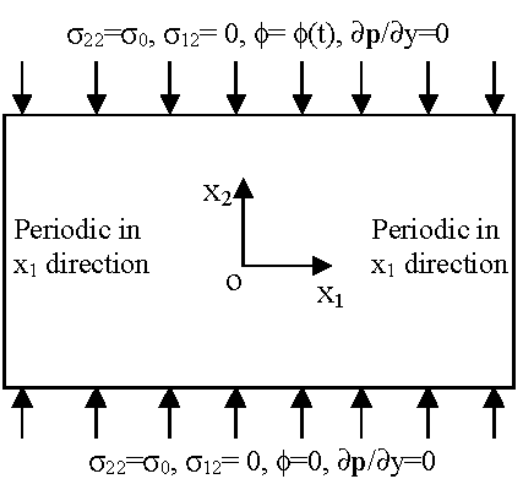

(c)

Fig. 1. (a) Contour of free energy $\mathrm{W}^{\prime}$ for $\mathrm{BaTiO}_{3}$. (b) Profile of energy $\mathrm{W}^{\prime}$. (c) Computational cell and boundary conditions.

We introduce defects to enable domain nucleation. Specifically, we pick arbitrary spots (with area ratio $1 \%$ ) in our computational cell and change the material parameters to $a_{1}^{\prime}=-0.001, a_{2}^{\prime}=0.009, a_{3}^{\prime}=0.0003, a_{4}^{\prime}=0.0021$ and $a_{5}^{\prime}=0$ at these points (the elastic constants $c_{1}^{\prime}, c_{2}^{\prime}$, and $c_{3}^{\prime}$, and the coupling parameters $b_{1}^{\prime}, b_{2}^{\prime}$, and $b_{3}^{\prime}$, and consequently the spontaneous polarization and strains, are left unchanged). In this case, the defects are assumed to have the same spontaneous polarization and strains. Consequently, the barriers between energy wells are about $10 \%$ of those of $\mathrm{BaTiO}_{3}$. This is a simple "soft spot" defect model that allows us to see domain nucleation. It is an interesting question to relate these to actual nucleation mechanisms. ${ }^{7}$

Since polarization is the primary order parameter in this model, we first integrate Eq. (2) from time $t^{n}$ to $t^{n+1}=t^{n}+\Delta t$ to compute new polarizations. Then Eq. (3) is solved for new voltage and Eq. (4) is solved (respecting the compatibility 
of strains) to obtain new strains using the polarization values at time $t^{n+1}$. The normalized time step $\Delta t^{\prime}=1$ is found to result in convergent solutions. All the variables are defined as double precision to minimize numerical errors.

\section{RESULTS}

We first verify our model by simulating a cubic to tetragonal (square to rectangle) phase transformation phenomenon. As shown in Fig. 2(a), initially we put some randomly distributed polarization (noise) in the computational cell at time $t^{\prime}=0$. Because we have a negative $a_{1}^{\prime}$, the cubic phase of the material is not stable. Thus the material transforms to the low energy state and the polarization goes toward one of the four energy wells of the tetragonal phase. The interaction between polarization dipoles through the electric field and the stress field results in the appearance of domains. At $\mathrm{t}^{\prime}=3.6 \times 10^{3}$ shown in Fig. 2(b), one $180^{\circ}$ and four $90^{\circ}$ domain walls develop. However, the domain structure is not stable yet; at $t^{\prime}=7.2 \times 10^{3}$ it can be seen in Fig. 2(c) that two of the four $90^{\circ}$ domain walls are about to disappear. Ultimately, a domain structure that includes one $180^{\circ}$ and two $90^{\circ}$ domain walls remain at $\mathrm{t}^{\prime}=28.8 \times 10^{3}$ as in Fig. $2(\mathrm{~d})$. This remains stable for subsequent time. One can in fact show that this is a local minimum of Eq. (1).

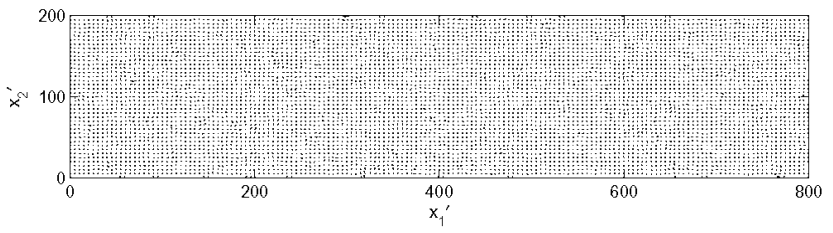

(a)

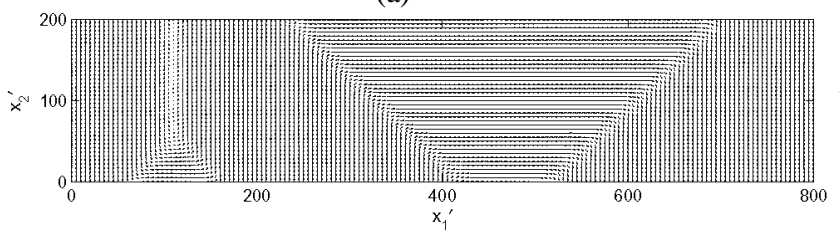

(c)

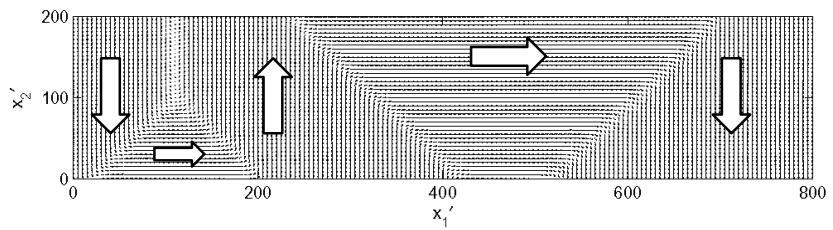

(b)

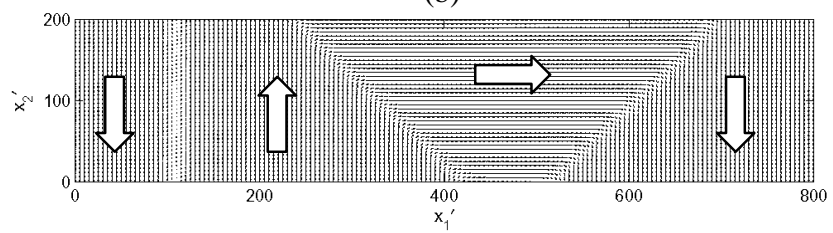

(d)

Fig. 2. Simulation results of cubic to tetragonal phase transformation: (a) $t^{\prime}=0$, (b) $t^{\prime}=3.6 \times 10^{3}$, (c) $t^{\prime}=7.2 \times 10^{3}$, (d) $t^{\prime}=28.8 \times 10^{3}$. The orientations of the domains are indicated with large arrows.

If we start from some different initial noise, the final domain picture would be different from the one shown in Fig. 2(d). But we find in repeated simulations that the stable state consists of $180^{\circ}$ and head-to-tail $90^{\circ}$ domain walls.

To further demonstrate the model's capability, we present the results of stress-induced domain switching in Fig. 3. In this case, we start from a domain structure including two $90^{\circ}$ domain walls at $\mathrm{t}^{\prime}=0$ as shown in Fig. 3(a), and gradually increase the compressive stress on the top and bottom of the computational cell. Fig. $3(\mathrm{~b})$ indicates that at $\mathrm{t}^{\prime}=7.2 \times 10^{3}$ the domain walls have moved toward the center to let the domains reorient in the horizontal direction. The compressive stress is kept constant at $\sigma_{0}{ }^{\prime}=-0.3$ after $\mathrm{t}^{\prime}=14.4 \times 10^{3}$, as illustrated in Fig. 3(c). The history of average polarization (average electrical charge per unit area on top or bottom) and the history of average strain $\left(\varepsilon_{22}\right)$ in the computational cell are plotted in Fig. 3(d). We see that domains finish switching around $t^{\prime}=10 \times 10^{3}$ and thereafter the average polarization remains approximately zero (indicating that all the domains are finally oriented horizontally) while the average strain approaches a constant value of $-0.725 \%$. Here, the average strain is due to both the phase transition and the applied compressive stress. Therefore, its absolute value is larger than the spontaneous strain at zero stress $\left(\varepsilon_{a}=-0.44 \%\right)$.

As another illustrative example, we simulate the domain switching process driven by an electric field. In this case, we start from the same domain structure with only two $90^{\circ}$ domain walls shown in Fig. 3(a), then slowly decrease the voltage on the top of the computational cell (so that the electric field increases). Figs. 4(a) and (b) reveal that the energetically favored vertically oriented domain in the center grows at the cost of the horizontally oriented domains. The applied electric field versus time curve is shown in Fig. 4(c). In Fig. 4(d), the average polarization and average strain are plotted against electric field. It is apparent that domain switching finishes when electric field is approximately equal to 
$1 \times 10^{-3}$. From then on, the average polarization and average strain do not change too much although the electric field still increases. The fact that the average strain does not change too much shows that ordinary electrostriction strain is not as large as the strains caused by domain switching.

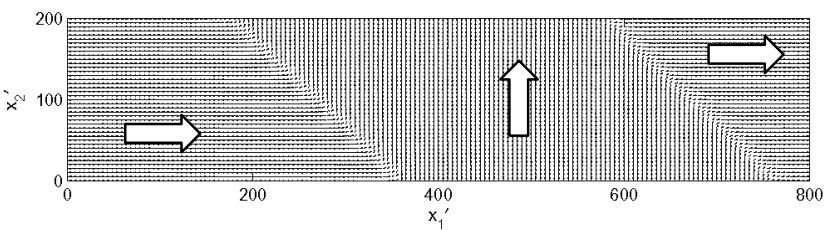

(a)

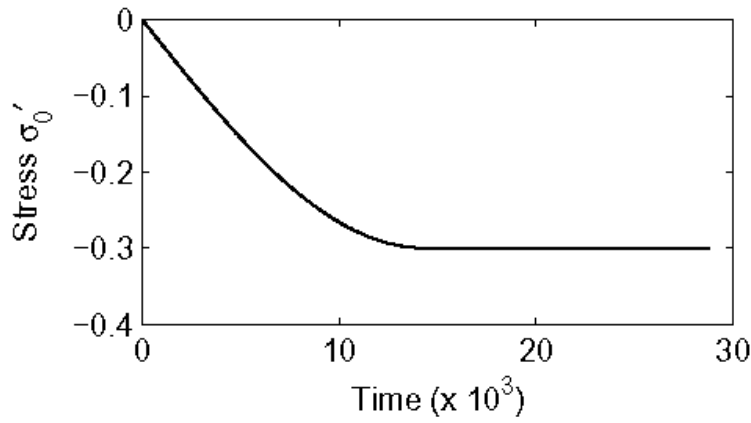

(c)

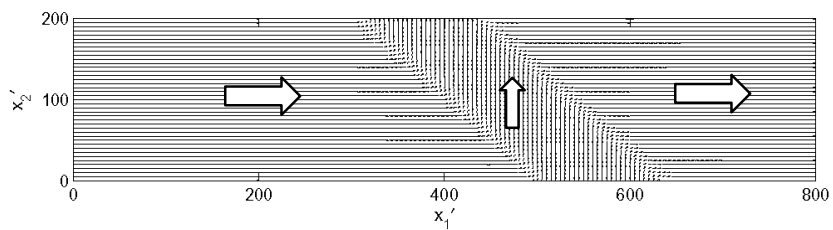

(b)

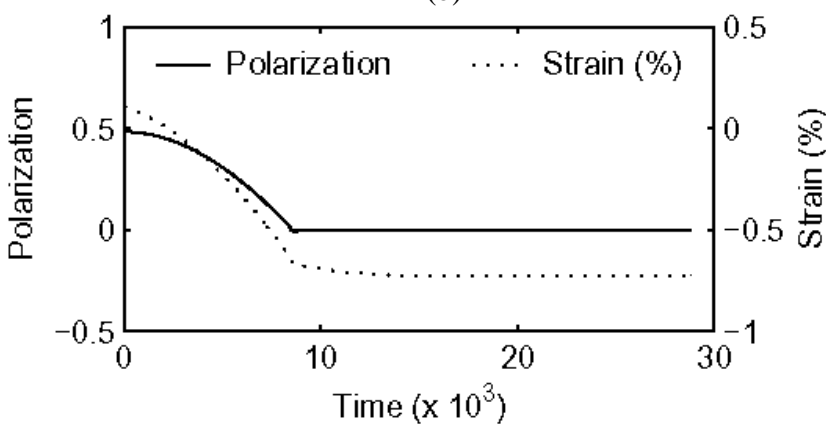

(d)

Fig. 3. Stress induced domain switching: (a) $\mathrm{t}^{\prime}=0$, (b) $\mathrm{t}^{\prime}=7.2 \times 10^{3}$. (c) Applied stress vs. time. (d) Polarization and strain history.

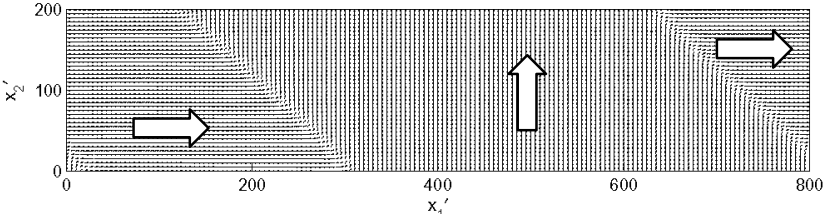

(a)

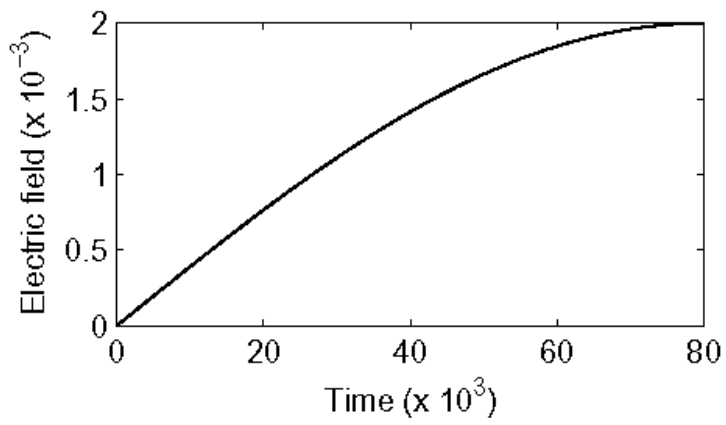

(c)

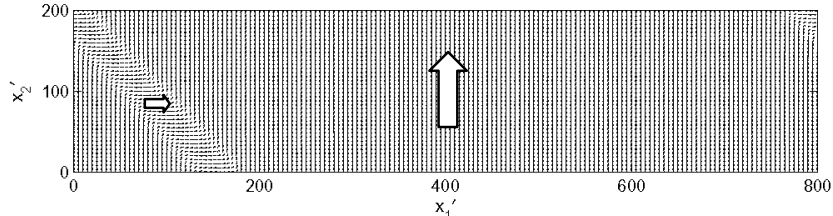

(b)

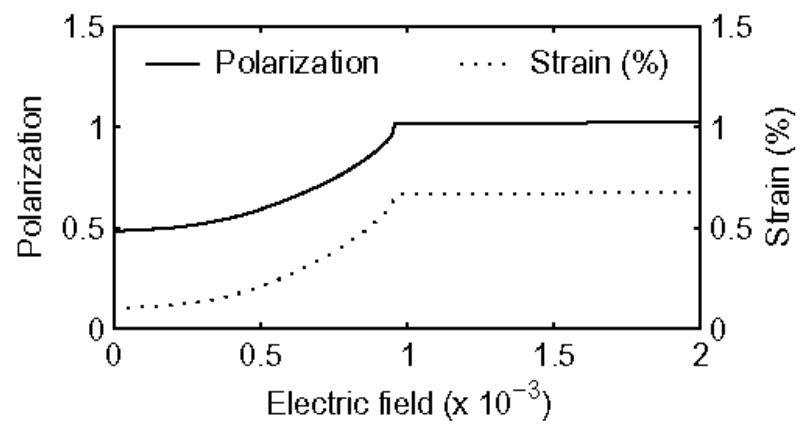

(d)

Fig. 4. Electric field driven domain switching: (a) $t^{\prime}=15 \times 10^{3}$, (b) $t^{\prime}=25 \times 10^{3}$. (c) Electric field vs. time. (d) Polarization and strain vs. electric field.

We now turn to the experiments by Burcsu, Zhang, Ravichandran and Bhattacharya under constant stress and cyclic electric field. ${ }^{4,5}$ We introduce defects as described earlier to enable nucleation. We start with a specimen consisting of a single domain ( $[0 \overline{1}]$ phase), and load it mechanically with a compressive stress $\sigma_{0}^{\prime}=-0.1$ until equilibrium is established at time $t^{\prime}=0$ as shown in Fig. 5(a). Subsequently the electric field is increased, and a new domain nucleates with two $180^{\circ}$ domain walls (see Fig. $5(\mathrm{~b})$ when $\mathrm{t}^{\prime} \approx 110 \times 10^{3}$ ). This domain continues to grow as the electric field is increased till it completely drives out the original domain and the specimen is fully in the [01] phase. Eventually the electric field 
reaches its peak and begins to decrease. A domain with [10] phase and subsequently another with $[0 \overline{1}]$ phase nucleates as shown in Fig. 5(c) at $t^{\prime} \approx 752 \times 10^{3}$. As the electric field further decreases, the domain walls propagate as in Fig. 5(d) and finally a single domain in the $[0 \overline{1}]$ phase is obtained. If we continue to run another cycle, the above sequence repeats. We do not observe any difference between the result in the first cycle from that in the second cycle. The polarization versus electric field (polarization hysteresis) and strain versus electric field (butterfly hysteresis) curves are shown in Fig. 5(f) and are in good qualitative agreement with experimental observations. ${ }^{4}$

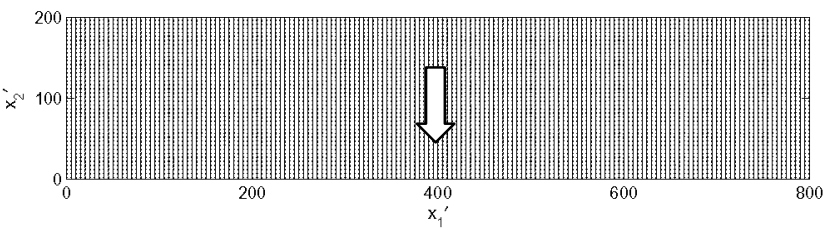

(a)

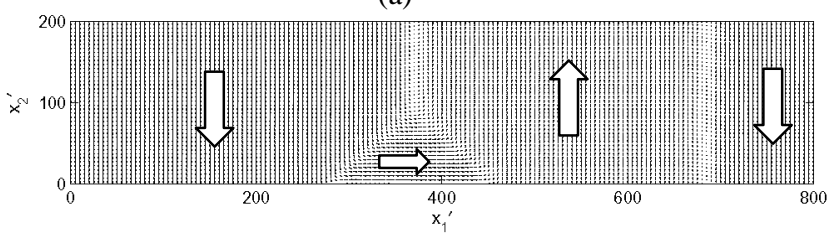

(c)

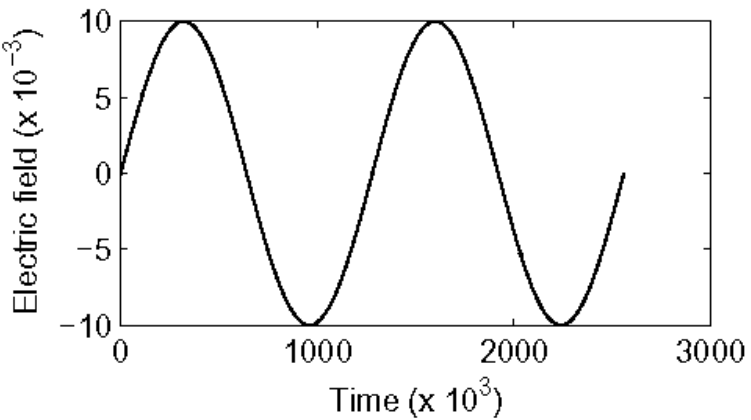

(e)

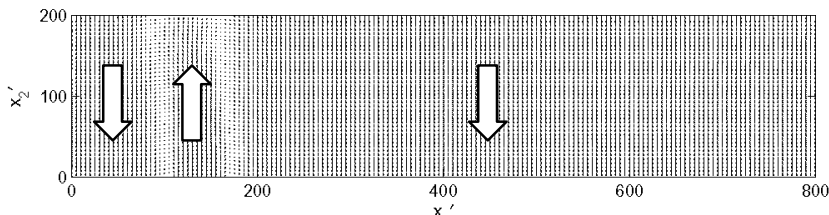

(b)

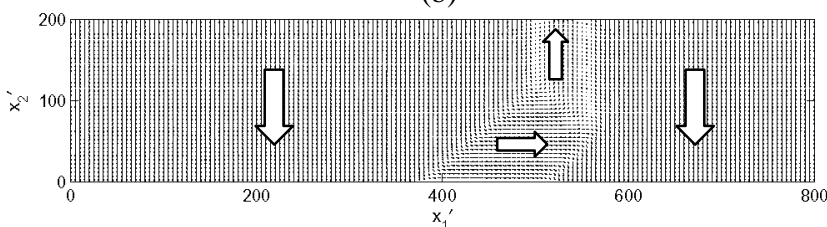

(d)

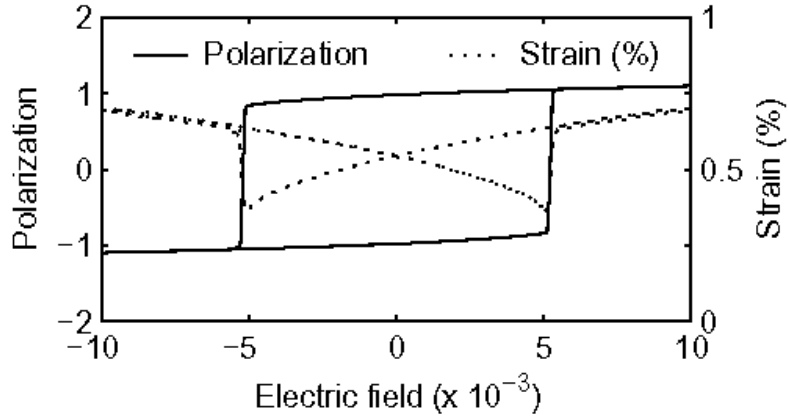

(f)

Fig. 5. Domain switching under combined electromechanical loads when $\sigma_{0}{ }^{\prime}=-0.1$ : (a) $\mathrm{t}^{\prime}=0$, (b) $\mathrm{t}^{\prime} \approx 110 \times 10^{3}$, (c) $\mathrm{t}^{\prime} \approx 752 \times 10^{3}$, and (d) $\mathrm{t}^{\prime} \approx 753 \times 10^{3}$. (e) Electric field vs. time. (f) Polarization hysteresis and butterfly hysteresis.

Figs. 6(a)-(d) show the results of the same experiment, but for a larger value of applied compressive stress, $\sigma_{0}{ }^{\prime}=-0.27$. Compared to the previous case at $\sigma_{0}{ }^{\prime}=-0.1$ (Fig. 5), we see more $90^{\circ}$ domain switching (and more [10] or [10] phases). It is clear that compressive stress favors $90^{\circ}$ domain switching.

The combination of $90^{\circ}$ switching and $180^{\circ}$ switching is helpful for large actuation strains. It has been theoretically predicted ${ }^{6}$ and experimentally observed ${ }^{4}$ that the actuation strain for $\mathrm{BaTiO}_{3}$ can be as large as $1.1 \%$. However, optimal electromechanical loads are needed to reach such an output strain. To systematically investigate the influence of electromechanical loads on the hysteresis curves, we conducted a series of simulations at different stress levels. The results are summarized in Fig. 7(a) for polarization hysteresis and in Fig. 7(b) for butterfly hysteresis. Fig. 7(a) shows that the coercive electric field (the field strength necessary to bring the polarization to zero) decreases with the compressive stress magnitude. It implies that depolarization is easier under large compressive stresses. In Fig. 7(b), on the other hand, we notice that the actuation strain increases with the stress level. This is because more $90^{\circ}$ domain switching happens under a large compressive stress. The simulation results in Figs. 5-7 are consistent with the experimental measurements by Burcsu, Zhang, Ravichandran and Bhattacharya. ${ }^{4,5}$ 
In ongoing and future work we use this model to investigate the influence of different loading, the role of frictional contact on damage and the interaction between domain walls and grain boundaries. We also plan to use this model as a design tool for a novel class of microactuators. ${ }^{8}$

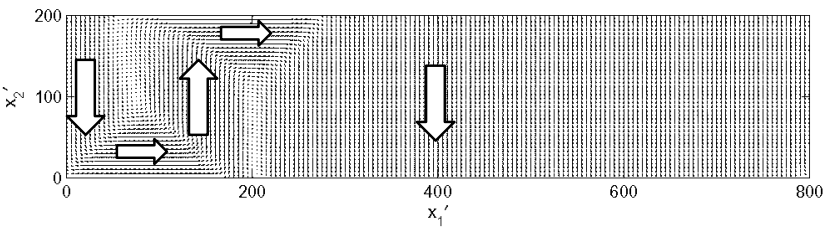

(a)

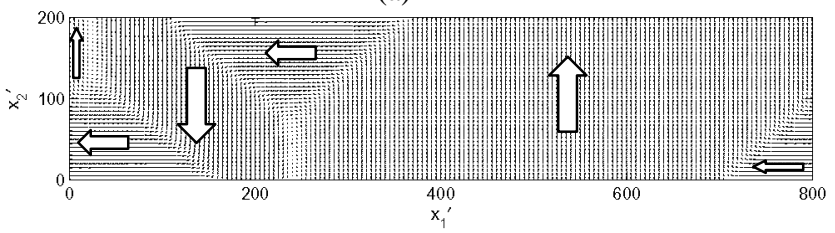

(c)

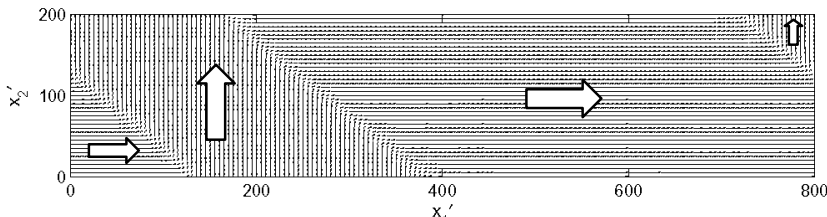

(b)

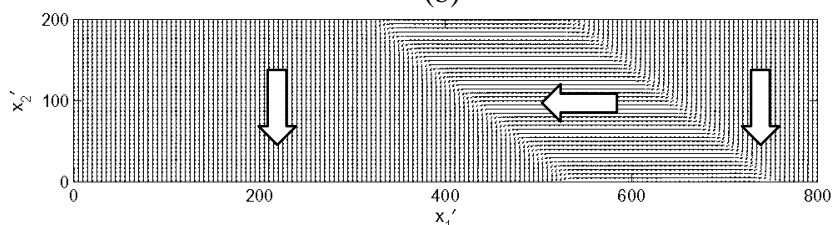

(d)

Fig. 6. Domain switching under electromechanical loads when $\sigma_{0}{ }^{\prime}=-0.27$ : (a) $t^{\prime} \approx 79 \times 10^{3}$, (b) $t^{\prime} \approx 83 \times 10^{3}$, (c) $t^{\prime} \approx 720 \times 10^{3}$, (d) $t^{\prime} \approx 736 \times 10^{3}$.

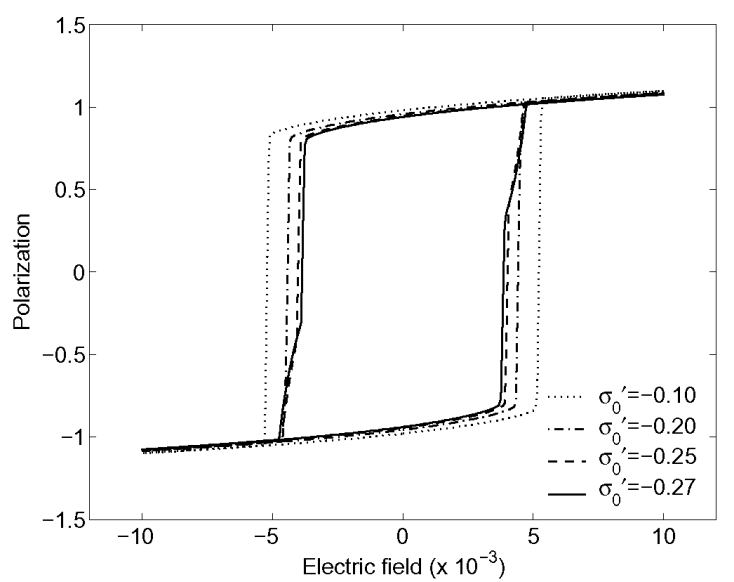

(a)

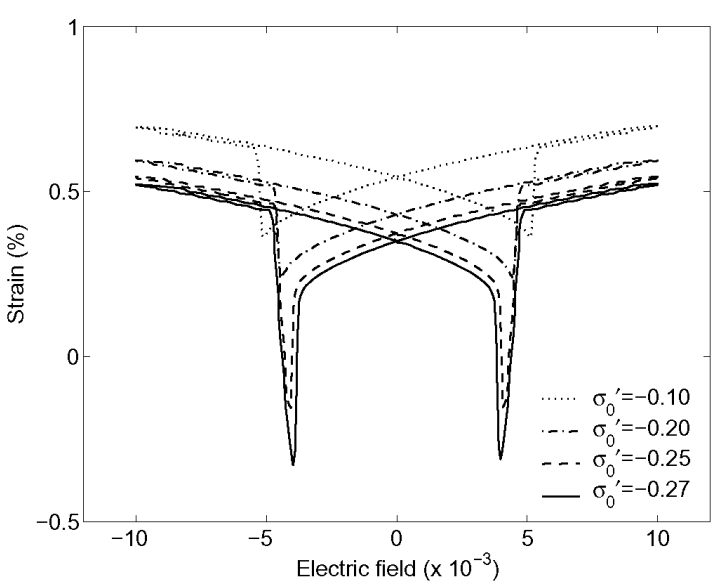

(b)

Fig. 7. Hysteresis curves at different compressive stress levels: (a) polarization hysteresis and (b) butterfly hysteresis.

\section{CONCLUSIONS}

A computational model that takes into account domain interactions by means of electric field is presented. It is shown that the model predicts not only cubic to tetragonal phase transformation phenomenon but also domain reorientations (domain switching in tetragonal phase) under combined electromechanical loads. Nucleation of domains and movement of domain walls are also successfully modeled. Simulated macroscopic behaviors in terms of polarization hysteresis and butterfly hysteresis agree well with experimental observations. The model can be used to assist in designing novel thin film ferroelectric microactuators.

\section{ACKNOWLEDGEMENT}

We are glad to acknowledge numerous discussions with Prof. G. Ravichandran and the financial support of the Army Research Office through the MURI grant No. DAAD19-01-1-0517. 


\section{REFERENCES}

1. Cao W, Cross LE, "Theory of tetragonal twin structures in ferroelectric perovskites with a first-order phase transition," Phys. Rev. B 44(1), 5-12, 1991.

2. Hu HL, Chen LQ, "Three-dimensional computer simulation of ferroelectric domain formation," J. Am. Ceram. Soc. 81(3), 492-500, 1998.

3. Ahluwalia R, Cao W, "Computer simulations of domain pattern formation in ferroelectrics," AIP conference proceedings, edited by H. Krakauer, 582, 185-190, 2001.

4. Burcsu E, Ravichandran G, Bhattacharya K, "Large strain electrostrictive actuation in barium titanate," Appl. Phys. Lett. 77(11), 1698-1700, 2000.

5. Zhang R, Ravichandran G, Bhattacharya $\mathrm{K}$, "Electromechanical characterization and domain visualization during large electrostriction in ferroelectrics," SPIE's 10th Annual International Symposium on Smart Structures and Materials, San Diego, California, USA, 2-6 March, 2003.

6. Shu YC, Bhattacharya K, "Domain patterns and macroscopic behavior of ferroelectric materials," Phil. Mag. B 81(12), 2021-2054, 2001.

7. Zhang W, Bhattacharya $\mathrm{K}$, "A computational model for phase transformation and large strain actuation of ferroelectric materials," in preparation.

8. http://www.femuri.caltech.edu. 